\title{
Contingent Claim Pricing Using the Cauchy Probability Distortion Operator under Simple Transformation
}

\author{
Bright O. Osu and Godswill U. Achi
}

\begin{abstract}
The problem of pricing contingent claims has been extensively studied for non-Gaussian models, and in particular, Black- Scholes formula has been derived for the NIG asset pricing model. This approach was first developed in insurance pricing where the original distortion function was defined in terms of the normal distribution.

This approach was later studied to compare the standard Black-Scholes contingent pricing and distortion based contingent pricing. In this paper, we aim at using distortion operators by Cauchy distribution under a simple transformation to price contingent claim. We also show that we can recuperate the Black-Sholes formula using the distribution.
\end{abstract}

Index Terms-Wang transformation, NIG and cauchy distribution under a simple transformation, distortion operator, contingent pricing.

\section{INTRODUCTION}

It is a well-known fact that the returns of most financial assets have semi-heavy tails and the actual kurtosis is higher than that of a normal distribution. A form of insurance risk pricing based on a normal-based distortion operator has been proposed [1]. This pricing principle is consistent with the financial theory of Gaussian option pricing as shown in [2]. It is shown that the celebrated Black Scholes formula can be recuperated through the distortions operator under the assumption of a normal model for asset prices [3]. The Wang not only possesses various desirable properties as a pricing method but also has a sound economic interpretation. Among them the most striking result in the transform, is that it is consistent with Buhlmann's economic premium principle. The pricing principle approach of Wang must be somehow modified in order for it to capture the non- Gaussian feature of market prices. In recent years, several non-Gaussian distributions have been proposed in order to better the model asset prices. One of such models is the NIG process. Under non-Gaussian assumptions for asset returns, markets are incomplete and there are many equivalent martingale measures. This implies that the arbitrage -free price of contingent claims is not unique. Among this family of equivalent measures, one can find subclasses for which explicit formulae can work. One of such subclasses is the so called mean-correcting equivalent martingale [4]. Moreover, a Black-Scholes type formula can be worked out in this case. This is an interesting analogue to the situation found in the Brownian model for which the celebrated Black-Scholes formula for contingent claims was first developed, Instead, in

Manuscript received July 1, 2012; revised December 3, 2012.

The authors are with the Department of Mathematics, Abia State University, Uturu, P. M. B.2000. Nigeria. (e-mail:megaobrait@yahoo.com). this paper we aim at pricing contingent, using the Cauchy distribution operators (under a transformation). We also recover Black-Scholes formula. This paper is organized as follows:

In section 2, we present a brief summary of results about the Wang and NIG family distribution and the corresponding non-Gaussian financial theory. In section 3 we introduce the Cauchy [under a simple transformation] distortion operator and discuss some of their properties and features. In section 4, we show how this new operator is consistent with standard non-Gaussian financial theory by recuperating the Black-Scholes type formula. Section 5 finally concludes.

II. Summary of Result of Wang Distortion AND Nig FAMILY OF DISTRIBUTION AND THE CORRESPONDING NON-GAUSSIAN FINANCIAL THEORY.

Let $X$ be a random variable representing a financial (insurance) risk and let $F_{X}$ and $S_{X}$ be its distribution and survival function respectively. The premium (price) associated with this position is

$$
\pi(X)=\int g\left(S_{X}(x)\right) d x
$$

where $g$ is an increasing differentiable function with $0<\mathrm{g}<1$, for all $x[5]$.

Moreover, this function is such that $g(0)=0$ and $g(1)=1$. Equation (1) shows that the premium function $\pi$ can be seen as a corrected mean under a new density measure given by;

$$
\pi(X)=\int x g^{\prime}\left(S_{X}(x)\right) d F_{X}(x)=E^{\mu}[X]
$$

where the $E^{\mu}$ denotes expectation under the density measure $\mu$. Wang [6] Proposed $S$ the following class of distortion function based on the normal distribution in order to price insurance and financial risks

$$
g_{\alpha}(u)=\phi\left(\phi^{-1}(u)+\alpha\right)
$$

where $\phi$, is the standard normal cumulative distribution function. Hamada and Sherris [2] shows that the distortion (3) is consistent with Black-Sholes formula. Let us consider the following price Kernel associated with distortion in (3).

$$
H[X=h(z), \alpha]=\int g_{\alpha}\left(S_{X}(x)\right) d x
$$

where $h$ is a continuous, positive and increasing function. For a normal random variable $Z$,

$$
H[X=h(x), \alpha]=E[h(z+\alpha)] .
$$


In the Standard Black-Scholes model, asset prices follow a geometric Brownian motion with

so that

$$
\frac{d X(t)}{X(t)}=\mu d t+\sigma d W_{t},
$$

$$
X_{T}=\left(X_{0} e^{\left(\mu-\frac{\sigma^{2}}{2}\right) T+\sigma W_{T}}\right)
$$

A standard European call option has pay off at maturity $T$ and we can write $f(z)$, where $z$ is a standard normal random variable

$$
f(z)=\left(X_{0} e^{\left(\mu-\frac{\sigma^{2}}{2}\right) T+\sigma \sqrt{T z}-k}\right)
$$

Applying the relation (kernel), we have

$$
\begin{gathered}
H(C(T, k) ;-\alpha)=E[f(z+\alpha)] \\
=\int_{-\infty}^{\infty}\left(X_{0} e^{\left(\mu-\frac{\sigma^{2}}{2}\right) T+\sigma \sqrt{T \alpha}+\sigma \sqrt{T z}-k}+\frac{1}{\sqrt{2 \pi}} e^{\frac{-z^{2}}{2} d z}\right) \\
=X_{0} e^{\mu T-\sigma \sqrt{T \alpha}}\left[1-\phi\left(z_{\text {min }}+\sigma \sqrt{T)}\right]-k\left[1-\phi\left(z_{n}\right)\right]\right. \\
=X_{0} e^{\mu T-\sigma \sqrt{T \alpha}} \phi\left(-z_{\text {min }}+\sigma \sqrt{T}\right)-K \phi\left(-z_{\text {min }}\right) .
\end{gathered}
$$

Calibrating Wang's discounted certainty equivalent to the underlying security price using $\alpha=\frac{\left(\mu-r_{c}\right)}{\sigma} \sqrt{T}$ gives;

$$
\begin{gathered}
e^{-r_{c} T} H(C(T, k),-\alpha)=X_{0} \phi\left(\frac{\ln \left(\frac{X_{0}}{k}\right)+\left(r_{c}+\frac{\sigma^{2}}{2}\right)^{T}}{\sigma \sqrt{T}}\right) \\
-e^{-r_{c} T} k \phi\left(\frac{\ln \left(\frac{X_{0}}{k}\right)+\left(r-\frac{\sigma^{2}}{2}\right)}{\sigma \sqrt{T}}\right) .
\end{gathered}
$$

The generalized version of the distortion in (3) is based on $a$

Normal inverse Gaussian rather than a normal distortion.

Definition 2: Let $\phi^{N I G}$ denotes the NIG cumulative distribution function $N I G(\sqrt{\alpha \delta},-\beta \sqrt{\delta / \alpha}, \sqrt{\alpha \delta}, 0)$. We define the NIG distortion as

$$
g_{\alpha} \beta, \delta, \theta(u)=\phi^{N I G}\left(\phi^{N I G^{-1}}(u)+\emptyset\right)
$$

These can be calibrated just like in Wang's distortion. One interesting feature of this new distortion is that since it is based on a skewed distribution, the underlying probabilities are distorted asymmetrically at the tails. In Wang's distortion, it is the same way because of the symmetry of the normal distribution.

Proposition 1: consider the $N I G$ distortion $g_{\alpha}, \beta, \delta, \theta$ defined in (4). Let $Z$ be a random variable with distribution given by $N I G\left(g_{\alpha}, \beta, \delta, \mu\right)$ and let $X=h(z)$ be the transformation through a continuous, positive, and increasing function $h$, then

$$
H[X, \theta]=\int g_{\alpha, \beta, \delta, \theta}\left(S_{X}(x)\right) d x=E[h(z+\theta \sqrt{\delta / \alpha}]
$$

For the proof see [6].

Now

$$
S_{X}(t)=p\left[\frac{z-\mu}{\sqrt{\delta / \alpha}}>\frac{h^{-1}(t)-\mu}{\sqrt{\delta / \alpha}}\right]
$$

Because of the symmetry property of the parameter $\beta$ in proposition $1, S_{X}(t)=\phi^{N I G}\left[\frac{\mu-h^{-1}(t)}{\sqrt{\delta / \alpha}}\right]$.

Applying the distortion, we have

$$
g_{\alpha, \beta, \delta, \theta}\left(S_{X}(t)\right)=\phi^{N I G}\left(\frac{\mu-h^{-1}(t)}{\sqrt{\delta / \alpha}}+\theta\right) .
$$

Considering the following exponential NIG asset price model

$$
S_{t}=S_{0} e^{Z_{t}}, t>0
$$

where $z_{t}$ is a $\left(f_{i}, p\right)^{2}-N I G$ levy process with parameters $[\alpha, \beta, \delta, \mu]$. Then the $\left(f_{i}, p\right)$ random variable $S_{T}$ is the price of the security at time $\mathrm{T}$ and it can be written as $S_{T}=h\left(z_{t}\right)$ for a function $h(u)=S_{0} e^{u}$ and a random variable $z_{T}$ with distribution $\operatorname{NIG}\left(\alpha, \beta, \delta_{T}, \mu_{T}\right)$.

If we apply proposition 1 , we have that

$$
H\left[S_{T},-\theta\right]=S_{0} e^{\mu T-\theta \sqrt{\mu / \alpha}-\delta_{T}\left[\sqrt{\alpha^{2}}-(\beta H)^{2}-\sqrt{\alpha^{2}}-\beta^{2}\right]},
$$

where

$$
\theta=\frac{\mu-r-\delta \sqrt{\alpha^{2}-(\beta+1)^{2}}-\sqrt{\alpha^{2}-\beta^{2}}}{\sqrt{\delta / \alpha}} .
$$

In other words, under the $N I G$ distortion with a value of $\theta$, the price $S_{T}$ evolves like a risk-neutral asset [7].

Hence,

$$
\begin{aligned}
H\left[f\left(S_{T}, k\right):-\theta\right]=S_{0} & \int_{\ln \left(\frac{k}{s_{0}}\right)}^{\infty} e^{z} \operatorname{nig}\left(z, \alpha, \beta, \delta T\left[\mu+\theta^{*}\right] T d z\right. \\
& -\int_{\ln \left(\frac{K}{S_{0}}\right)}^{\infty} \operatorname{nig}\left(z, \alpha, \beta, \delta T,\left[\mu+\theta^{*}\right] d z .\right.
\end{aligned}
$$

This implies that the price of a standard European pay-off evaluated with the pricing kernel associated to the NIG distortion with a parameter $\theta^{*}$ is given by

$$
\begin{array}{r}
e^{r t} H\left[f\left(S_{T}, k\right):-\theta\right] \\
=S_{0} N I G\left(\ln \frac{k}{S_{0}}, \alpha, \beta+1, \delta T\left[\mu+\theta^{*}\right] T\right) \\
-K e^{-r T} N I G\left(\ln \frac{k}{S_{0}}, \alpha, \beta, \delta T,\left[\mu+\theta^{*}\right] T\right) .
\end{array}
$$

Equation (9) is the Black-Scholes type formula at time $t=0[1]$

This shows that the NIG distorted pricing kernel with parameter $\theta$ reduces to the Black-Scholes type formula under the mean correcting Martingale measure. 


\section{The CAUChy Distribution Under A SimPle TRANSFORMATION}

A random variable $S$ follows a Cauchy distribution under a simple transformation of dividing through by a constant with parameter vector $(a, b)$, in symbolic notation $S \sim$ Cauchy $(a, b)$, if its probability density function is

$$
f\left(S_{t} ; a, b\right)=\frac{b \varrho}{\pi}\left(\frac{1}{b^{2}+(s-a)^{2}}\right), \quad-1<S_{t}<1,
$$

where $\varrho$ is the stabilization term [8]. The formula for the stabilization term is given as;

$$
\varrho=\frac{\pi}{2 \tan ^{-1}\left(\frac{1-a}{b}\right)} \text {. }
$$

The pdf (10) can therefore be written as

$$
\begin{gathered}
f(s ; a, b)=\frac{1}{2 \tan ^{-1}\left(\frac{1-a}{b}\right)}\left(\frac{1}{b^{2}+(s-a)^{2}}\right),-1<S_{t}<1, \\
a<1, b \geq 1 .
\end{gathered}
$$

Note that $a$ and $b$ are ordinary parameter of location and scales. Under this transformation, the variance $S$ is obtained by;

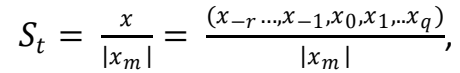

by letting $\left|x_{m}\right|=\max \left|x_{-r}, \ldots x_{0}, \ldots x_{q}\right|, x \in R,-r<x<$ $q: r, q \in R$ [9]. $R$ is the real space without the points, $-\infty$ and $\infty \mathrm{X}$ is a Cauchy random variable.

The first four moments of this probability density function are obtained as;

$$
\begin{aligned}
& E(s)=\frac{1}{2 \tan ^{-1}\left(\frac{1-a}{b}\right)}\left[\begin{array}{c}
\frac{1}{2}\left[\ln \left(1+a^{2}+b^{2}-2 a\right)-\ln \left(1+a^{2}+b^{2}+2 a\right)\right] \\
+\frac{a}{b}\left(\tan ^{-1}\left(\frac{1-a}{b}\right)-\tan ^{-1}\left(\frac{-1-a}{b}\right)\right)
\end{array}\right], \\
& E\left(s^{2}\right)=\frac{1}{\tan ^{-1}\left(\frac{1-a}{b}\right)}\left[\begin{array}{c}
1+a\left[\ln \left(1+a^{2}+b^{2}-2 a\right)-\ln \left(a^{2}+b^{2}\right)\right] \\
+\frac{a^{2}-b^{2}}{b}\left(\tan ^{-1}\left(\frac{1-a}{b}\right)-\tan ^{-1}\left(\frac{-a}{b}\right)\right)
\end{array}\right],
\end{aligned}
$$

$E\left(s^{3}\right)=$

$$
\frac{1}{2 \tan ^{-1}\left(\frac{1-a}{b}\right)}\left[\begin{array}{c}
4 a+\frac{3 a^{2}-b^{2}}{2}\left[\ln \left(1+a^{2}+b^{2}-2 a\right)-\ln \left(1+a^{2}+b^{2}+2 a\right)\right] \\
+\frac{a^{2}-3 a b^{2}}{b}\left(\tan ^{-1}\left(\frac{1-a}{b}\right)-\tan ^{-1}\left(\frac{-1-a}{b}\right)\right)
\end{array}\right]
$$

and

$$
\begin{aligned}
& E\left(s^{4}\right) \\
& =\frac{1}{2 \tan ^{-1}\left(\frac{1-a}{b}\right)}\left[\begin{array}{c}
\frac{1}{3}+a-b^{2}+3 a^{2}+2 a\left(a^{2}-b^{2}\right) \ln \left(1+a^{2}+b^{2}-2 a\right) \\
+b\left(b^{2}-6\right)\left(\tan ^{-1}\left(\frac{1-a}{b}\right)\right)-2 a\left(a^{2}-b^{2}\right) \ln \left(a^{2}-b^{2}\right)+ \\
b\left(b^{2}-6\right)\left(\tan ^{-1}\left(\frac{-a}{b}\right)\right)
\end{array}\right]
\end{aligned}
$$

From which we obtain the expression for the skewness and kurtosis as:

$$
\operatorname{skew}(a, b)=\frac{\varphi^{(2)}(a, b)+\emptyset^{2}(a, b)}{\left[\varphi^{(1)}(a, b)+\emptyset^{(1)}(a, b)\right]^{\frac{3}{2}}}
$$

where

$$
\begin{gathered}
\varphi^{(1)}(a, b)=1+\ln \left[\frac{\left(1+a^{2}+b^{2}-2 a\right)^{a}}{a^{2}+b^{2}}\right] \\
\emptyset^{(1)}(a, b)=\frac{a^{2}-b^{2}}{b}\left[\tan ^{-1}\left(\frac{1-a}{b}\right)+\tan ^{-1}\left(\frac{1-a}{b}\right)\right] \\
\varphi^{2}(a, b)=4 a+\left[\ln \frac{\left(1+a^{2}+b^{2}-2 a\right)^{\frac{3 a^{2}-b^{2}}{2}}}{1+a^{2}+b^{2}-2 a}\right] \\
\emptyset^{2}(a, b)=\frac{a^{3}-3 a b^{2}}{b}\left[\tan ^{-1}\left(\frac{1-a}{b}\right)+\tan \left(\frac{1-a}{b}\right)\right] \\
\operatorname{Kurt}(a, b)=\frac{\varphi^{(3)}(a, b)+\emptyset^{3}(a, b)}{\left[\varphi^{(1)}(a, b)+\emptyset^{(1)}(a, b)\right]^{2}}
\end{gathered}
$$

where

$$
\begin{array}{r}
\varphi^{3}(a, b)=\frac{1}{3}+a-b^{2}+3 a^{2} \\
+\left[\ln \frac{\left(1+a^{2}+b^{2}-2 a\right)^{\frac{2 a\left(a^{2}-b^{2}\right)}{2}}}{a^{2}+b^{2}}\right] \\
\emptyset^{(3)}(a, b)=b\left(b^{2}-6\right)\left[\tan ^{-1}\left(\frac{1-a}{b}\right)+\tan ^{-1}\left(\frac{a}{b}\right)\right] .
\end{array}
$$

From the above four expressions for the moments of $\mathrm{S}$, we have ; $\lim _{a \rightarrow 0, b \rightarrow 0} \gamma_{1}(a, b)=0$ and $\lim _{a \rightarrow 0, b \rightarrow \infty} \gamma_{1}(a, b)=$ 0 .

Hence the limiting values of the kurtosis as tends to zero and $\mathrm{b}$ tends to infinity are given by $\lim _{a \rightarrow 0, b \rightarrow 0} \gamma_{2}(a, b)=$ $1 / 3$ and $\lim _{a \rightarrow 0, b \rightarrow \infty} \gamma_{2}(a, b)=-1$

Moreover, given $\xi=\frac{1}{\tan ^{-1}\left(\frac{1-a}{b}\right)}$, then the Cauchy distribution under simple transformation can be written as

$$
\begin{gathered}
C(a, b)=\xi \frac{1}{b^{2}+(S-a)^{2}}=\xi\left\{b^{2}+(S-a)^{2}\right\}^{-1} \\
\quad=\xi b^{-2}\left\{1+\left(\frac{S-a}{b}\right)^{2}\right\}^{-1}
\end{gathered}
$$

$$
\begin{array}{r}
=\xi b^{-2} \exp \left\{-\log \left[1+\left(\frac{S-a}{b}\right)^{2}\right]\right\} \\
\leq \xi b^{-2} \exp \left\{-\left(\frac{S-a}{b}\right)^{2}\right\} .
\end{array}
$$

where $a, b$ are obtained by applying the maximum likelihood estimation method as [8]:

$$
a=\sum_{i=0}^{n}\left(S_{i}+1\right) \pm \sqrt{\left(S_{i}+1\right)^{2}-4\left(S_{i}+b\right)^{2}},
$$

and 


$$
b=\sum_{i=0}^{n} \sqrt{(1-a)\left(S_{i}-a\right)} .
$$

The NIG distribution as well as the Cauchy distribution are infinitely divisible and the Cauchy distribution appears as the opposite limit as $a \rightarrow 0$ for the inverse Gaussian distribution;

$$
N I G(x, a, \beta, \mu, \delta) \sim k|x|^{1.5} \exp \left(\frac{-a \delta}{\delta}|x|+\frac{\beta}{\delta} x\right)
$$

when $|x| \rightarrow 0$. We therefore assume herein that a random variable $X \sim(C(a, b)$.

\section{Probability Distortion Approach And Change of MEASURE}

Let $W=\left\{W_{t}, t \geq 0\right\}$ be a standard Brownian motion and let $I=I_{t}, t \geq 0$ be an IG process with parameters $=1, b=$ $\delta \sqrt{\alpha^{2}-\beta^{2}}$, with $\alpha>0,-\alpha<\beta<\alpha$ and $\delta>0$; then it can be shown that the stochastic process $X_{t}=\beta \delta^{2} I_{t}+\delta W_{I_{t}}$ is an NIG process with parameters $\alpha, \beta$ and $\delta$ [10].

Let $F(t, x)=e^{x-1 / 2^{t}}$ be the future price and let $X_{t}=B_{t}$. Then we formally obtain

$$
\begin{gathered}
d F(t, x)=\frac{\partial F}{\partial t} d t+\sum_{i=1}^{d} \frac{\partial F}{\partial x} d X_{t}^{i}+\sum_{i, j=1}^{d} \frac{\partial^{2} F}{\partial x^{2}} d X_{t}^{i} d X_{t}^{j} \\
=e^{B_{t}-1 / 2^{t}}
\end{gathered}
$$

This implies that

$$
d F\left(t, B_{t}\right)=F\left(t, B_{t}\right) d B_{t}
$$

Consider the stochastic exponential

$$
\varepsilon(B)_{t}=e^{B_{t}-1 / 2^{t}}
$$

We see that it has the stochastic exponential

$$
d \varepsilon(B)_{t}=\varepsilon(B)_{t} d B_{t}
$$

This relation can be treated as a stochastic differential equation with a solution delivered by (22). Thus

$$
\varepsilon(X)_{T}=\varepsilon(0) e^{\beta \delta^{2} I_{t}+\delta W_{I_{t}}-\frac{1}{2} t} .
$$

Putting (24) in a broader context we consider now the process

$$
Z_{t}=\exp \left\{\int_{0}^{t} b(t, w) d B_{t}-1 / 2 \int_{0}^{t} b^{2}(s, w) d s\right\}
$$

where $b=(b(t, w))_{t \geq 0}$ is a non-anticipating process with

$$
P\left(b^{2}(s, w) d s<\infty\right)=1, t>0 .
$$

Setting

$$
Y_{t}=\int_{0}^{t} b(s, w) d B_{t}-1 / 2 \int_{0}^{t} b^{2}(s, w) d s
$$

and $F(y)=e^{y}$, we can use ito's formula to see that that process $Z=\left(Z_{t}\right)_{t \geq 0}$ (the Girsanov exponential) has the stochastic differential

$$
d Z_{t}=Z_{t} b(t, w) d B_{t}
$$

Lemma 1: Let $\left\{X_{t}\right\}_{t \geq 0}$ be a drifting Brownian motion process where $X_{t}=\beta \delta^{2} I_{t}+\delta W_{I_{t}}$ and $\left\{W_{t}\right\}_{t \geq 0}$ is a $\mathbb{P}_{-}$ Brownian motion and $\beta$ and $\delta$ are constants. Then a measure under which $\left\{X_{t}\right\}_{t \geq 0}$ is a martingale is given as

$$
W_{I_{t}}^{(L)}=W_{I_{t}}+\beta \delta I_{t}
$$

Proof : Taking $\vartheta=\frac{\beta \delta^{2}}{\delta}$, under the probability measure $\mathbb{P}^{(L)}$ of Girsonov's theorem we have that the process $\left\{W_{I_{t}}^{(L)}\right\}_{0 \leq I_{t} \leq I_{T}}$, defined by

$$
W_{I_{t}}^{(L)}=W_{I_{t}}+\int_{0}^{I_{t}} \vartheta_{s} d s
$$

is a standard Brownian motion [11]. Thus

$$
\begin{aligned}
W_{I_{t}}^{(L)} & =W_{I_{t}}+\int_{0}^{I_{t}} \beta \delta d \\
& =W_{I_{t}}+\stackrel{\beta}{\delta} \delta I_{t},
\end{aligned}
$$

is a Brownian motion and $X_{t}=\delta W_{I_{t}}$ is a scaled Brownian. Notice that

$$
\begin{gathered}
\mathbb{E}^{\mathbb{P}}\left[X_{t}^{2}\right]=\mathbb{E}^{\mathbb{P}}\left[\beta^{2} \delta^{4} I_{t}^{2}+2 \beta \delta^{2} I_{t}+\delta^{2} W_{I_{t}}^{2}\right] \\
=\beta^{2} \delta^{4} I_{t}^{2}+\delta^{2} I_{t},
\end{gathered}
$$

where as

$$
\mathbb{E}^{\mathbb{P}^{(L)}}\left[X_{t}^{2}\right]=\mathbb{E}^{\mathbb{P}^{(L)}}\left[\delta^{2}\left(W_{I_{t}}^{(L)}\right)^{2}\right]=\delta^{2} I_{t}
$$

If we apply the kernel, the distortion $g_{(a, b)}$ on $g_{x}$, we have

$$
\varepsilon(X)_{T}=\varepsilon(0) e^{\beta \delta^{2} I_{t}+\delta W_{I_{t}}-\frac{1}{2} t} .
$$

a standard European call option has pay-off at maturity

$$
\mathfrak{C}(\varepsilon(T), K)=\left(\varepsilon\left(S_{T}\right)-K\right)_{+}, \quad 0 \leq t \leq T .
$$

where $S_{T}$ is the price of the security at maturity and we can write this as $f(Z)$ where $Z$ is a Cauchy random variable and

$$
f(Z)=\left(\varepsilon(0) e^{\beta \delta^{2} I_{t}+\delta \sqrt{T} Z-\frac{1}{2} t}-K\right)_{+}
$$

Theorem 1: Let $S=x-\frac{1}{2} T$, where $x=\beta \delta^{2} I_{t}+\delta \sqrt{T} Z$ and let $\mathfrak{C}: \mathbb{R} \rightarrow \mathbb{R}$ satisfy for $\alpha>0$

$$
\int_{-\infty}^{+\infty} e^{-\alpha S^{2}}|\mathfrak{C}(S)| d S<\infty .
$$

Then

$$
\begin{gathered}
H(\mathfrak{C}(\varepsilon(T), K))=e^{\beta \delta^{2} I_{T}-\frac{1}{2} T-\left(\frac{\pi}{2} b^{2} \delta \sqrt{T}\right)^{2}} C\left(S-b^{2} \delta \sqrt{T}\right)- \\
K C\left(\frac{S}{b}\right) .(33)
\end{gathered}
$$


Proof : Applying the relation to Wang distortion formula on (32)given $\alpha=b^{-2}$, we have:

$$
\begin{aligned}
& H(\mathfrak{C}(\varepsilon(T), K))=\xi b^{-2} \int_{-\infty}^{+\infty}\left(\varepsilon(0) e^{\beta \delta^{2} I_{t}+\delta \sqrt{T} Z-\frac{1}{2} T}\right. \\
& -K)_{+} \exp \left\{-\left(\frac{Z-a}{b}\right)^{2}\right\} d Z \\
& =\xi b^{-2} \int_{-\infty}^{+\infty}\left(\varepsilon(0) e^{S-a}-K\right) \exp \left\{-\left(\frac{Z}{b}\right)^{2}\right\} d Z \\
& =\xi b^{-2} \int_{-\infty}^{S} e^{S-a-\left(\frac{Z}{b}\right)^{2}} d Z-K \xi b^{-2} \int_{-\infty}^{\frac{S}{b}} e^{-\bar{Z}^{2}} d \bar{Z} \\
& =\xi b^{-2} \int_{-\infty}^{S} e^{S-a-\left(\frac{Z}{b}\right)^{2}} d Z-K C\left(\frac{S}{b}\right) \\
& =\xi b^{-2} e^{\beta \delta^{2} I_{T}-\frac{1}{2} T} \int_{-\infty}^{S} e^{\delta \sqrt{T Z}-\left(\frac{Z}{b}\right)^{2}} d Z-K C\left(\frac{S}{b}\right) \\
& =\xi b^{-2} e^{\beta \delta^{2} I_{T}-\frac{1}{2} T} \int_{-\infty}^{S} e^{-\frac{1}{b^{2}}\left(Z^{2}-b^{2} \delta \sqrt{T} Z\right)} d Z-K C\left(\frac{S}{b}\right) \\
& =\xi b^{-2} e^{\beta \delta^{2} I_{T}-\frac{1}{2} T} \int_{-\infty}^{S} e^{-\frac{1}{b^{2}}\left(Z-b^{2} \delta \sqrt{T}\right)^{2}-\left(\frac{\pi}{2} b^{2} \delta \sqrt{T}\right)^{2}} d Z \\
& -K C\left(\frac{S}{b}\right) \\
& =\xi b^{-2} e^{\beta \delta^{2} I_{T}-\frac{1}{2} T-\left(\frac{\pi}{2} b^{2} \delta \sqrt{T}\right)^{2}} \int_{-\infty}^{\left(S-b^{2} \delta \sqrt{T}\right)} e^{-\frac{1}{b^{2}} \bar{Z}^{2}} d \bar{Z} \\
& -K C\left(\frac{S}{b}\right) \\
& =e^{\beta \delta^{2} I_{T}-\frac{1}{2} T-\left(\frac{\pi}{2} b^{2} \delta \sqrt{T}\right)^{2}} C\left(S-b^{2} \delta \sqrt{T}\right)-K C\left(\frac{S}{b}\right) .
\end{aligned}
$$

Remark: if $b=\sqrt{2}$ the result is as in [2].

\section{TIME - VARYING DRIFT AND VOLATILITY FOR THE SECURITY PRICE}

Consider the Cauchy distortion under a transformation defined in [2]. Let $Z$ be the random variable with distribution given by $C(a, b)$ and let $X=h(Z)$ be a transformation through a continuous, positive and increasing function $h$.

We now have to study how this distortion affects an exponential Levy model for assets prices and in particular if there is a value of $\theta$ such that discounted asset prices behave like risk-neutral prices.

Let us consider the following exponential Cauchy (under a transformation) asset price model

$$
S_{t}=S_{0} e^{z_{t}}, \quad t>0
$$

where $Z_{t}$ is a $\left(f_{t}, p\right)$ Cauchy-Levy (under transformation) process with parameters $(a, b)$.

Then the $\left(f_{t}, p\right)$-random variable $S_{t}$ is the price of the security at time $\mathrm{T}$ and it can be written as $S_{T}=h\left(Z_{T}\right)$.

For a function $h(U)=S_{0} e^{u}$ and a random variable $Z_{T}$ with distribution $C(a, b)$, then we have;

$$
H\left[S_{T},-\theta\right]=E[f(Z+\theta)]
$$

$$
=\left(\varepsilon(0) e^{\beta \delta^{2} I_{t}+\delta \sqrt{T} Z-\frac{1}{2} t}-K\right)_{+} .
$$

Definition 2 : $\operatorname{Let}\left(\varphi, F\left(F_{t}\right), p\right)$ be a filtered probability space. An adapted cadlag R-valued process, $X=\{X(t)\}_{t \geq 0}$ with $\quad x(0)=0$ is a Cauchy-levy process under transformation if $x(t)$ has independent and stationary increment distributed as Cauchy $(; a, b)$

Now we choose $\theta$ such that the discounted price process

$$
\left\{\exp (-(r-q) t) s_{t}, t \geq 0\right\}
$$

is martingale ie

$$
S_{0}=\exp (-(r-q) t) E^{\theta}\left[S_{t}\right]
$$

where expectation is taken with respect to the law with density $f_{t}^{(\theta)}(x), q$ is the rate of yield of compound dividends per annum and $\mathrm{r}$ the interest rate. Let $\emptyset(u)=E\left[\exp \left(u i X_{i}\right)\right]$ denote the characteristics function of $X_{i}$. Then from (35), in order to let the discounted prices process be a Martingale, we need to have as in [10];

$$
\exp (r-q)=\frac{\emptyset(-i(\theta+1))}{\emptyset(-\theta)}
$$

It is easy to see that

$$
\theta=\left(\frac{\mu-r+q-2(a-b)}{2(a-b)}\right)
$$

such that

$$
S_{T}=W_{t}+\left(\frac{q+\mu-r-2(a-b)}{2(a-b)}\right)
$$

and so

$$
\zeta_{t} \triangleq \frac{d \mathbb{Q}^{\zeta}}{\mathbb{Q}} \mid \Im_{t}=\exp \left\{2(a-b) X_{t}-2(a-b) t\right\}
$$

Now,

$$
E_{t}=E_{0} \exp \left\{2(a-b) S_{t}-2(a-b) t+(r-\mu) t\right\} .
$$

The pay-off of the contract will be $C_{\tau}=E_{\tau}-K$. Expressing $E_{\tau}$ as a function of $X_{\tau}$ gives (36) and so using that $\left\{X_{t}\right\}_{t \geq 0}$ is a $\mathbb{Q}$ - Brownian motion gives the fair value of $K$ as

$$
K=E^{\mathbb{Q}}\left[E_{\tau}\right]=e^{(r-\mu)} E_{0} .
$$

Hence calibrating Cauchy discounted certainty equivalent to the underlying security price using (37) gives

$$
\begin{gathered}
e^{-r_{C} T} H(\mathfrak{C}(\varepsilon(T), K))=\varepsilon(0) C\left(\frac{\ln \left(\frac{S_{0}}{K}\right)+\mu-r+q+2(a-b) T}{2(a-b) \sqrt{T}}\right)- \\
K C e^{-r_{C} T}\left(\frac{\ln \left(\frac{S_{0}}{K}\right)+\mu-r+q-2(a-b) T}{2(a-b) \sqrt{T}}\right),
\end{gathered}
$$

which is the Black-Scholes price of the call option at time 0 . 
This demonstrates that Cauchy's distortion function approach recovers the Black-Scholes price of a European call options.

\section{CONCLUSION}

The most common way to estimate the value of options is to use the Black-Sholes formula. If the price changes of a security are log normally distributed, the Black-Sholes formula provides the theoretical price of the so called European options on that security. Unfortunately, there is overwhelming evidence that price changes are not log-normally distributed. Instead, security price changes have what is often called fat-tails. They also exhibit skewness. Fat-tails can be modelled with so called stable distribution which is also referred to as Levy distributions and Levy-Pareto distribution. A Gaussian distribution is a special case of stable distribution. The Cauchy distribution is another well-known example of a stable distribution. In fact, the Gaussian and Cauchy distribution are the only two stable distributions for which closed form mathematical formula exist and it is consistent with the behaviour we observe in real capital markets. Hence equation (35) demonstrates that Cauchy distortion function approach under a transformation recovers the Black-Scholes price of a European call option.

\section{REFERENCES}

[1] S.Wang, "A Class of Distortion operator for pricingFinance and Insurance risks,"Journal of Risk and Insurance, vol. 36 pp. 15-36, 2000 .

[2] M. Harmada and M. Sherries, "Contingent Claim Pricing using probability distortion operators," Methods from Insurance Risk pricing and their relationship to Financial theory and Applied Mathematical, Finance, 10, pp. 19-47, 2003.

[3] M. E. Yaari, "The Dual Theory of Choice under Risk," Econometrica, 55 pp. 95-115, 1987.

[4] R. C. Merton, "Option pricing when underlying Stocks returns are discontinuous," Journal of Financial Economics, vol. 3 pp. 125-144, 1976.
[5] E. Bolviken and F. E. Benth, "Quantification of Risk in Norwegian Stock via the normal inverse Gaussian distribution operators," SSM.com/SO13/delivery.CFM.nb1006275, vol. 300, pp. 2000. 1959.

[6] F. Godin, S. Mayoral, and M. Morales, "Contingent Claim Pricing Using a Normal Inverse Gaussian Probability Distortion Operator,' Working Paper no, CRM3290, 2000.

[7] J. F .C Kingsman and S. J. Taylor, Introduction to measure and probability, Cambridge University press, pp. 123-135, 1973.

[8] J. Ohakwe and B. O. Osu, "The Existence of the moments of the Cauchy distribution under a simple transformation of dividing with a constant," Theoretical Mathematics and application, vol. 1, no. 1, pp. 27-35, 2011.

[9] B. O. Osu and J. Ohakwe, "Financial risk assessment with Cauchy distribution under a simple transformation of dividing with a constant," Theoretical Mathematics and application, vol. 1, no. 1, pp. 73-89, 2011.

[10] W. Schoutens, Levy processes in Finance: Pricing Financial Derivatives, John wiley and Sons, Ltd., vol. 6, pp. 77-80, 2003.

[11] A. Etheridge, A course in Financial Calculus, University Press Cambridge CB2 2RU, UK. NY 10011: 4211, USA, vol. 5, pp. 123-126, 2002.

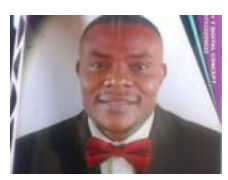

Bright O. Osu received a B.Sc. degree in Mathematics from Abia State University, Uturu, Nigeria in1998, an M.Sc. degree in Mathematics (Probability Theory) from University of Ibadan, Nigeria in 2003 and a PhD degree in Financial Mathematics from Abia State University, Uturu, Nigeria in 2008. He works in the Department of Mathematics Abia State University as a Senior Lecturer (Currently the Head of Department). His research interest includes: Stochastic Approximation, Mathematics of Finance and Probability Theory.

Godswill U. Achi obtained a B.Sc. degree in Mathematics from Abia State University, Uturu, Nigeria in 1994, an M.Sc. degree in Mathematics (Stochastic Control and Optimization) from Abia State University, Uturu,Nigeria in 2007. A Ph.D Student in Mathematics department, Abia State University, Uturu, Nigeria. He works in the department of Mathematics Abia State Polytechnic Aba,Nigeria as a Principal Lecturer ( Currently the Head of Department). His research interest includes; Stochastic control and Optimization, Mathematics of Finance and Probability Theory. 